\title{
ASSESSMENT OF MICROLEAKAGE AND MICROHARDNESS OF GLASS IONOMER CEMENT CONTAINING PROPOLIS: IN VITRO STUDY
}

\author{
Yomna El Ghazouly 1*, Aly Sharaf 2, Seham Hanafy, 3, Laila M. El Habashy 4.
}

\begin{abstract}
INTRODUCTION: Propolis is a natural product collected by bees. Contemporary dentistry has recently focused on it, due to its antimicrobial properties.

OBJECTIVES: To evaluate and compare the microleakage and microhardness of GIC containing EEP at 2 different concentrations to the conventional GIC.

MATERIALS AND METHODS: Two tests were assessed 1. Microleakage test :where 24 exfoliated primary teeth were divided randomly into three groups according to the restorative material used after standardized class V cavity preparation: (Group1: Conventional GIC, Group2: GIC containing 25\% EEP, and Group 3: GIC containing 50\% EEP), teeth were immersed in 2\% methylene blue solution for 24 hours, sectioned and examined under stereomicroscope. 2. Microhardness test: 8 specimens from each group (GIC, GIC 25\% EEP, and GIC 50\% EEP) were prepared using a mold of six mm in diameter and two mm in thickness. The hardness of the specimens was measured using Vickers Micro-hardness Tester.

RESULTS: There was no statistically significant difference in the microleakage scores between the three groups ( $\mathrm{p}=0.34$ ).The Microhardness values of the present study showed that GIC with 25\% EEP showed significantly higher values than both the conventional GIC and GIC with $50 \%$ EEP $(p<0.0001)$. There was no significant difference in the microhardness between the conventional GIC or GIC with 50\% EEP ( $>0.05$ )

CONCLUSION: The addition of EEP to GIC in either (25\%, 50\%) did not affect the microleakage of GIC. Addition of 25\% EEP increased the microhardness of the GIC while the addition of 50\% EEP did not affect the microhardness of GIC.

KEYWORDS: Ethanolic Extract of propolis, glass ionomer cement, Streptococcus mutants, microleakage, microhardness.

RUNNING TITLE: The effect of propolis on Microleakage and Microhardness of GIC.

1 Pediatric Dentistry and Oral Public Health Department, Faculty of Alexandria, Alexandria University, Egypt. 21526 Alexandria Egypt; yomna.maged@gmail.com

2 Professor of Pediatric Dentistry and Oral Public Health Department, Faculty of Dentistry, Alexandria University, Egypt. 21526 Alexandria Egypt; dr.alysharaf@hotmail.com

3 Professor of Biomaterial Department, Faculty of Dentistry, Alexandria University, Egypt. 2156 Alexandria Egypt; sehamahelmy@gmail.com

4 Pediatric Dentistry and Oral Public Health Department, Faculty of Dentistry, Alexandria University, Egypt. 2156 Alexandria Egypt;lmhabashy@hotmail.com
\end{abstract}

* Corresponding Author:

E-mail : yomna.maged@gmail.com.

\section{INTRODUCTION}

Dental caries in general and early childhood caries in particular is a major concern to the pediatric dentist. New methods in caries prevention are based on understanding the effect of antibacterial agents that can reduce the worldwide caries incidence (1). Recently, the direction toward natural products for medicinal purposes have gained much attention, as these natural products has proved to be effective with less toxic side effects, and thus can be used as an alternative source of treatment (2). Propolis is a natural gummy substance produced by honeybees as a result of the salivary enzymatic reaction of the bees on the plant exudates, it has been used widely in traditional medicine since ages ago mainly due to its high antioxidant properties (3).Studies have also shown other important properties in propolis such as antibacterial, antifungal, antiviral, anti-inflammatory, anesthetic, and ability to promote healing $(1,4,5)$.
The antibacterial activity of propolis on the oral microorganisms was proved by many studies (6) and now there are many oral products containing propolis in the form of toothpaste and mouth rinses. Bhuvnesh et al (7) assessed the antibacterial activity of Ethanolic and Water extract of propolis in two concentrations $5 \%$ and $20 \%$ in vitro. They found that the concentrations of $5 \%$ and $20 \%$ of EEP were effective against Streptococcus mutants and Lactic acidophilus, While the Water extract of $5 \%$ and $20 \%$ of propolis was effective only against Lactic acidophilus.

Since Glass ionomer cement has shown a variable antimicrobial activity in many studies in the literature (810),therefore to accentuate its antimicrobial effect the addition of other antimicrobial agents as Propolis was a point of the investigation by many studies. Nursen et al (12) concluded that GIC with 25\% and 50\% EEP showed anti-caries potential, as evident by reducing the incidence of caries and dental plaque accumulation in vivo, they explained their 
findings by two mechanisms associated with propolis properties:(i) antimicrobial activity against cariogenic bacteria ( Streptococcus mutants and Streptococcus sobrinus) and (ii) inhibition of glucosyltransferase enzymes (GTFs) activity $(13,14)$.

Other studies evaluated the effect of adding EEP on the mechanical properties of GIC. Altunsoy et al (11) showed that the microhardness of GIC containing 25\% of EEP increased more than GIC and also adding $50 \%$ of EEP to GIC increased the hardness of GIC more than adding 25\% EEP. The addition of (25\%, 50\%) EEP to GIC did not affect the microleakage.

Despite the previous efforts, the effect of Propolis on the physical properties of GIC is still not fully documented. Further studies are still needed to clarify the effect of GIC containing Propolis focusing on the physical properties of the restored teeth. Therefore, this study was planned to evaluate the physical/ mechanical properties of GIC containing Egyptian EEP that might affect the clinical performance of the restored teeth.

The null hypothesis of the current research claims that the addition of 2 different concentrations of Propolis (25 or 50\%) will not change the Microleakage and Microhardness of GIC.

\section{MATERIALS AND METHODS}

This experimental in-vitro study was conducted at the Pediatric Dentistry and Dental Public Health Department, and Dental Biomaterials Department after obtaining the approval of the research ethics committee (IORG008839), Faculty of Dentistry, Alexandria University. The study included two tests: I. Micro-leakage Test, II. Micro-hardness test.

Sample size calculation: The minimal sample size was calculated based on a previous study aimed to evaluate the effect of ethanolic extracts of propolis (EEP) using different proportions on microleakage and microhardness of GIC (13). By adopting a power of $80 \%$ to detect a standardized effect size in microhardness $\mathrm{d}=0.65$ (medium-sized standardized effect size), and level of significance $95 \%(\alpha=0.05)$, the minimum required sample size was found to be 8 specimens per group (number of groups $=3$ ) (sample size $=8 \times 3=24$ specimens) for micro hardness and 24 specimens for microleakage (Total sample size=48specimens) (15). The sample size does not need to be increased to control attrition (withdrawal) bias (16). The sample size was calculated using GPower version 3.1.9.2 (17).

Preparation of Propolis (EEP) (18): Ten grams of propolis (propolis powder Imtenan) was dissolved into $20 \mathrm{ml}$ of Ethanol (70\%W/V) forming the 50\% EEP. The filtering of the extract was done to remove rough particles. For preparing GIC with 50\% EEP powder and the liquid ratio were done according to manufacturer instruction $1: 1$ then $50 \%$ of the liquid was replaced by the prepared EEP. For preparing GIC with $25 \%$ EEP same procedure was done but only $25 \%$ of the GIC liquid was replaced by EEP $(12,18)$. This preparation was done in the Faculty of Pharmacy Pharos University.

Criteria of teeth selection

The posterior primary teeth were selected according to the following criteria:
1- Shed primary teeth.

2- No caries or previous filling or cracks.

3- No developmental anomalies

I-Microleakage Test

Sample selection: Fifty primary shed teeth were collected from Pediatric Dentistry clinics in public hospitals, Alexandria University, and private dental clinics. Teeth were hand scaled, cleaned using pumice with a rubber cup on low-speed handpiece then washed with water and stored in normal saline. The teeth were then carefully examined to discard those with any visible anatomical defects, cracks, or carious lesions (15). Twenty-four exfoliated primary teeth were selected that met the inclusion criteria $(19,20)$.

Cavity preparation: Class V cavities $(1.5 \mathrm{~mm}$ depth) were prepared on the buccal surfaces of the teeth $1 \mathrm{~mm}$ above the cementoenamel junction. Cavity standardization to have uniform dimensions, a window was cut in a stainless-steel band with the desired dimension of the cavity width and length (4mm wide x 2mm high) (21). A high-speed \#330 carbide bur (Komet, Germany) underwater coolant was used to prepare the desired cavity. The depth of the cavity was adjusted to $1.5 \mathrm{~mm}$. All Cavosurface angles were kept at right angles with no bevels. Then the cavity was rinsed with water for 10 seconds and gently air-dried for 5 seconds (19).

Sample grouping: The teeth were randomly assigned to 3 main groups ( $n=8 /$ each) according to the composition of the restorative material. Group1: high strength posterior restorative Glass ionomer cement (GIC) (Fuji IX GC Corporation Tokyo, Japan) Group 2: GIC containing 25\% of EEP, Group 3: GIC containing 50\% of EEP.

Procedure: All teeth were thermo-cycled for 500 cycles from $5{ }^{\circ} \mathrm{C}$ to $55{ }^{\circ} \mathrm{C}$ with $30 \mathrm{~s}$ dwell time, $20 \mathrm{~s}$ transfer time, corresponding to 6 months clinical use. Two layers of nail varnish were then applied over the teeth/ acrylic blocks leaving only $1 \mathrm{~mm}$ free around the tooth restoration border. The teeth were then immersed in 1\% methylene blue dye solution $(\mathrm{pH}=7)$ at $37^{\circ} \mathrm{C}$ for 24 hours and stored in the incubator (BST 5020, VEB MLW Dental Fabrik, Leipzig, Germany 1990), to allow diffusion of the dye into the gaps between the tooth and the restorative material. Teeth were then washed under tap water and sectioned buccolingual with low-speed water-cooled diamond saw. The sixteen specimens of each group were examined using a stereomicroscope (SZ1145TR, Olympus, Japan) at 20X magnification. An image of every section was captured using a video camera connected to the computer. Each section was examined for the presence of dye at all interfaces of the tooth. A microleakage score on the captured image was given by a single examiner who was blinded to the treatment and who was trained to the following scoring system: 0- No dye penetration, 1-Dye penetration through the cavity margin reaching the enamel tissue, 2-Dye penetration through the cavity margin reaching the dentine tissue, 3-Dye penetration through the cavity margin reaching the cavity floor portions (22).

II- Microhardness test: Eight discs shaped specimens from each material where group 1:GIC, group 2: GIC containing 25\% EEP,group3: GIC containing 50\% EEP, were prepared 
using a mold of six mm in diameter and two mm in thickness. After a complete setting reaction, the surfaces were covered by varnish, and all Specimens were stored in a humid environment at $37^{\circ} \mathrm{C}$ for 24 hours. The hardness of the specimens was measured using Vickers Microhardness Tester (402 MVD, Wolpert-Willison Instruments, Germany) with a Vickers diamond indenter and a 20x objective lens (11). A load of $300 \mathrm{~g}$ was applied to the surface of each specimen for 15 seconds, at least three indentations $200 \mu \mathrm{m}$ apart were done on each specimen. The diagonal length of the impressions was measured and hardness value (HV) was calculated according to the standard formula $\mathrm{H}=1.854 \mathrm{p} / \mathrm{d} 2$ (11). Statistical analysis

Normality was checked for Microhardness using the ShapiroWilk test. Variables were presented using Mean (SD) and 95\% Confidence interval while the Microleakage score was presented using percentages. One Way ANOVA was applied to compare the three groups regarding microhardness, followed by the Tukey post hoc test. Chi- Square test was used to assess differences concerning Microleakage. Statistically, the significant level was set at p-value $\leq 0.05$. Data were analyzed using IBM SPSS software package version 23.0 (Armonk, NY: IBM Corp)

\section{RESULTS}

The results of the present study showed that there were no statistically significant differences in the microleakage scores among any of the test groups GIC, GIC with 25\% EEP, and GIC with 50\% EEP ( $p=0.34)$. (Table 1, Figure1-4)

The Microhardness values of the present study showed that GIC with 25\% EEP (92.64 \pm 11.20$)$ showed significantly higher scores than GIC (50.60 \pm 13.86$)$, and GIC with $50 \%$ EEP $(47.08 \pm 8.00) \mathrm{p}<0.0001 *$. There was no significant difference in the microhardness scores between GIC and GIC with 50\% EEP. (Table 2, figure 5)

Table 1: Distribution of Microleakage scores among the study groups using Chi-Square test

\begin{tabular}{||c|c|c|c|}
\hline Microleakage scores & $\begin{array}{c}\text { Conventional } \\
\text { GIC } \\
(\mathbf{n}=\mathbf{1 6})\end{array}$ & $\begin{array}{c}\text { GIC with } \\
\mathbf{2 5 \%} \\
(\mathbf{n}=\mathbf{1 6})\end{array}$ & $\begin{array}{c}\text { GIC with } \\
\mathbf{5 0 \%} \text { EEP } \\
(\mathbf{n}=\mathbf{1 6})\end{array}$ \\
\cline { 2 - 4 } & \multicolumn{3}{|c|}{$\mathrm{n}(\%)$} \\
\hline Score 0 & $4(25 \%)$ & $7(43.8 \%)$ & $5(31.2 \%)$ \\
\hline Score 1 & $2(12.5 \%)$ & $2(12.5 \%)$ & $6(37.5 \%)$ \\
\hline Score 2 & $4(25 \%)$ & $2(12.5)$ & $3(18.8 \%)$ \\
\hline Score 3 & $6(37.5 \%)$ & $5(31.2 \%)$ & $2(12.5 \%)$ \\
\hline \multicolumn{3}{|c|}{ P value } & \multicolumn{3}{|c|}{0.34} \\
\hline
\end{tabular}



Figure 1: Distribution of Microleakage scores among the study groups.



Figure 2: Microleakage Scores in GIC Group: a. score 0- No dye penetration, b. score 1-Dye penetration through the cavity margin reaching the enamel tissue, c. score 2-Dye penetration through the cavity margin reaching the dentine tissue, d. score 3-Dye penetration reaching the cavity floor portions 


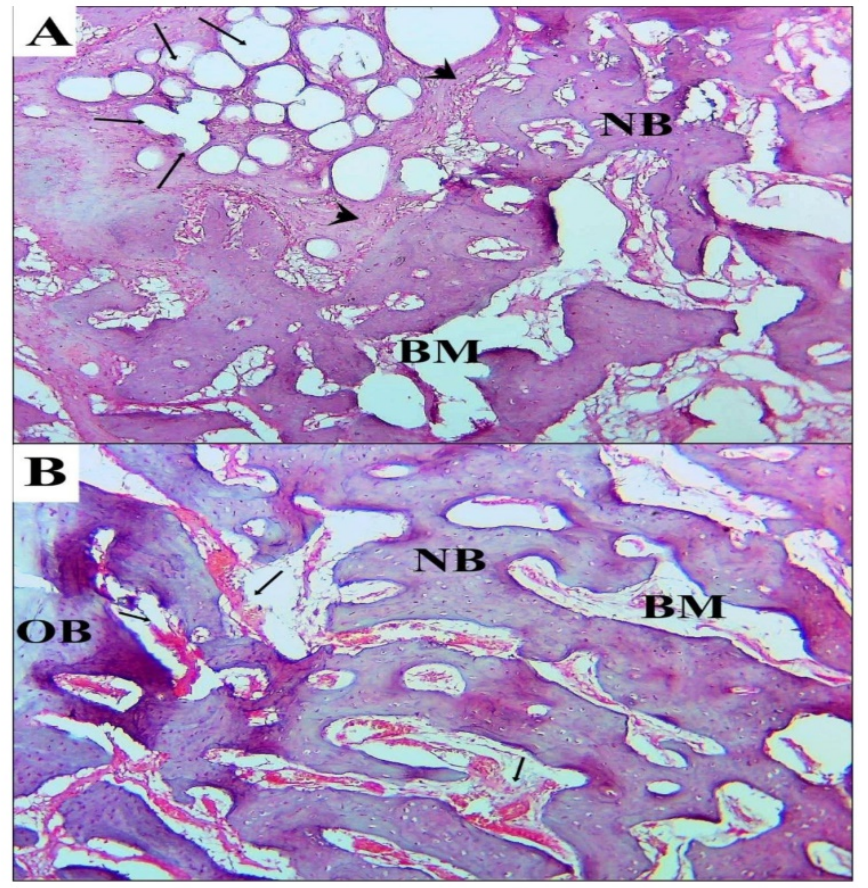

Figure 3: Microleakage Score in GIC with 25\% EEP Group: a. score 0 - No dye penetration, b. score 1-Dye penetration through the cavity margin reaching the enamel tissue, c. score 2-Dye penetration through the cavity margin reaching the dentine tissue, d. score 3Dye penetration reaching the cavity floor portions.

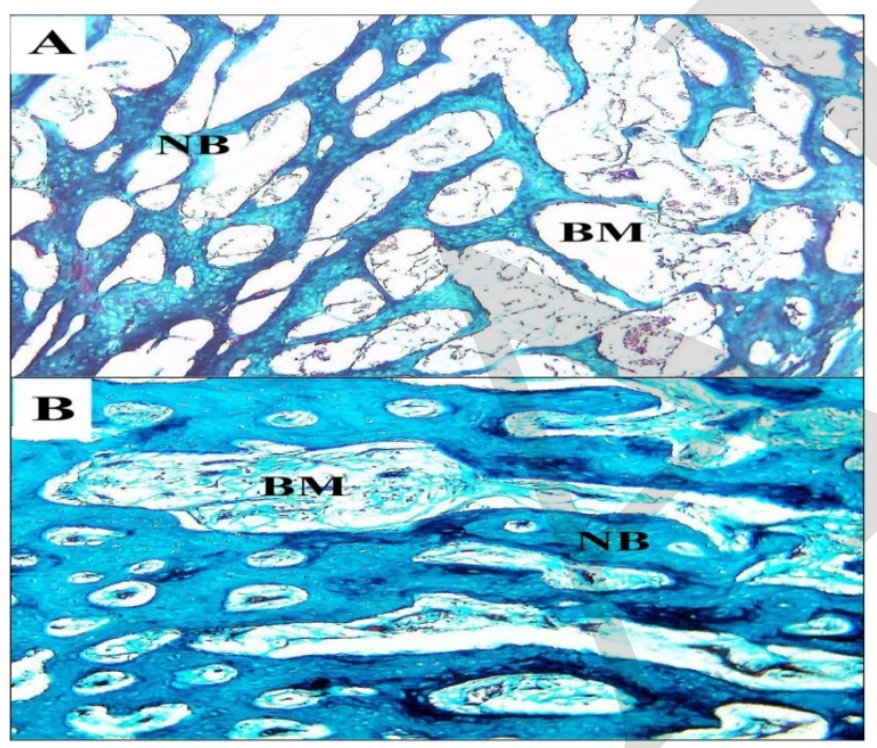

Figure 4: Microleakage Score in GIC with 50\% EEP Group: a. score 0- No dye penetration, b. score 1-Dye penetration through the cavity margin reaching the enamel tissue, c. score 2-Dye penetration through the cavity margin reaching the dentine tissue, d. score 3Dye penetration reaching the cavity floor portions
Table 2: Comparison of microhardness among the study groups using post hoc test

\begin{tabular}{|l|c|c|c||}
\hline & $\begin{array}{c}\text { Conventional GIC } \\
(\mathbf{n = 8 )}\end{array}$ & $\begin{array}{c}\text { GIC with } \\
\text { 25\% EEP (n=8) }\end{array}$ & $\begin{array}{c}\text { GIC with } \\
\mathbf{5 0 \%} \text { EEP(n=8) }\end{array}$ \\
\hline Mean (SD) & $50.60(13.86)$ & $92.64(11.20)$ & $47.08(8.00)$ \\
\hline 95\% CI & $39.01,62.19$ & $83.28,102.00$ & $40.38,53.77$ \\
\hline P value & \multicolumn{3}{|c|}{$<0.0001^{*}$} \\
\hline
\end{tabular}

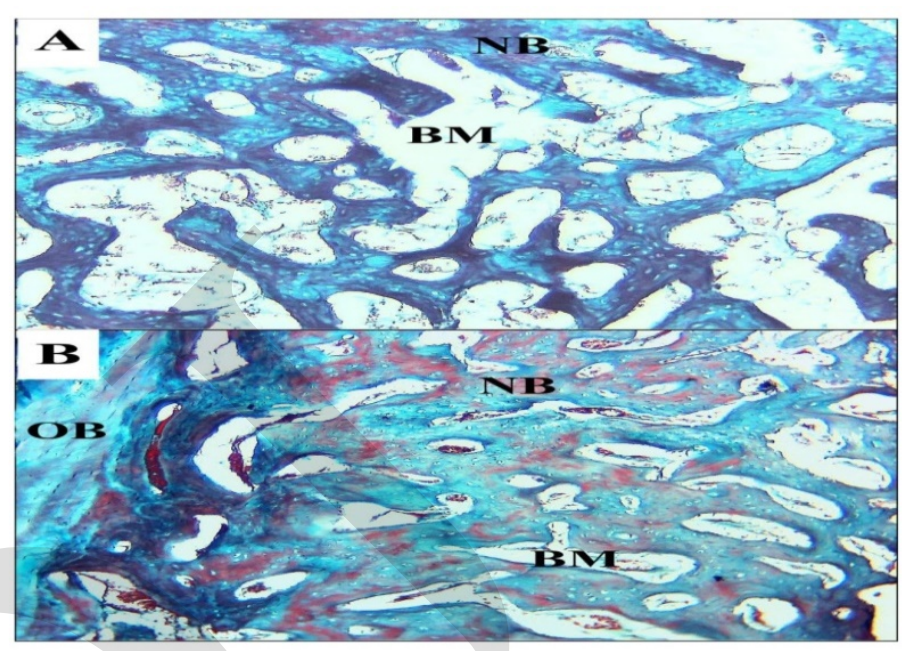

Figure 5: Mean and standard deviation of microhardness among the study groups.

\section{DISCUSSION}

Propolis has gained attention in the field of dentistry due to its antimicrobial effect especially on streptococcus mutant. Incorporation of propolis to GIC was done to benefit from the antimicrobial action of the propolis which would be of great importance especially in high- risk patients. However, studying the effect of propolis on the physical and mechanical properties GIC needs more investigation to exclude the possibility of lowering its strength or affecting its tooth bonding that would decrease the survival rate of the restoration and ultimately lead to failure. Therefore, this study aimed to evaluate the microhardness and microleakage of GIC containing Egyptian EEP at two concentrations 25\% and 50\% compared to conventional GIC.

In the present study two concentrations of propolis $(25 \%$ \& $50 \%$ ) were tested as in the literature review there was contra varsity between the effect of these two concentrations on GIC properties (23). The microleakage test was performed after all teeth were thermo-cycled for 500 cycles from $5{ }^{\circ} \mathrm{C}$ to $55^{\circ} \mathrm{C}$ with $30 \mathrm{~s}$ dwell time, $20 \mathrm{~s}$ transfer time, as this would correspond to six months clinical use and therefore would give more practical results on the intraoral performance of the restoration (24). Dye penetration test was used in this study as it is well known that materials with higher adhesion presents less microleakage scores, and thus better clinical performance (24).

The results of the present study showed that there was no significant difference between the microleakage scores of the GIC, GIC with $25 \%$ EEP or GIC with $50 \%$ EEP, indicating that the adhesion of GIC was not weakened by adding 
propolis at different concentrations. These findings were following Erdem et al (13) who showed that EEP addition to GIC did not affect the shear bond strength of GIC, also Altunsoy et al (11) concluded that 25\% and \%50\% of EEP did not affect GIC microleakage results.

The Microhardness values of the present study showed that GIC with 25\% EEP had significantly higher values than GIC, this might be due to the phenolic compounds present in EEP molecule resulting in the highly active material. A chelation reaction occurs between the phenolic part of EEP and GIC leading to a crosslinking process where more amount of acid reacts with the powder giving a more complex material with increased density and fewer voids (11).

Although these findings were in accordance to Altunsoy et al (11), however in this study the GIC with 50\% EEP showed significantly lower microhardness results than the GIC with $25 \%$ EEP this was inconsistent to their findings where the GIC with 50\% EEP showed significant higher microhardness than GIC with 25\% EEP. The difference in results between the two concentrations in the present study may be attributed to the higher water sorption of GIC with 50\% EEP than $25 \%$ EEP as proved by Troca VB, et al (23). The higher water sorption decreased the physical properties of GIC and ultimately gave lower microhardness results.

The physical performance of GIC with EEP (regarding the microhardness) in this study was inconsistent with Subramaniam et al (3) who found that by adding propolis to GIC, the solubility of the material significantly increased and the physical property significantly decreased in a period of seven days. They attributed their findings to the greater water sorption in GIC with EEP as proved by Troca VB, et al (23). In the present study, the GIC used was the new high strength posterior restorative material which differs in its physical property than the GIC. Since it contains higher filler particles that elicited higher translucency and higher reactivity of the material giving it more density and fewer voids, and thus although water sorption occurred, this did not decrease the physical property of the material. It can be assumed that different types of GIC might give different results after adding EEP depending mainly on the strength of the GIC used. Finally, it has to be noted that since adhesion of any restoration is mainly determined by shear bond strength and microleakage, therefore from the limitation of the present study was the absence of the shear bond strength test as it would have definitely high-lighted the effect of adding EEP on GIC adhesion in a more affirmative way.

The null hypothesis of this study was accepted in the microleakage test as there was no significant difference between the three test groups. However, the null hypothesis was rejected in the microhardness test as there was significant difference between the test groups.

\section{CONCLUSION}

Based on the findings of this study it was concluded that:

1. Adding propolis to GIC either as $25 \%$ or $50 \%$ did not affect the microleakage of the restoration.
2. The Microhardness of GIC was significantly increased by adding $25 \%$ EEP but on the contrary, adding 50\% of EEP to GIC did not increase the microhardness results.

\section{RECOMMENDATIONS}

More studies are needed to examine other physical properties of GIC containing propolis "EEP” before its clinical application.

\section{CONFLICT OF INTEREST}

The authors declare that they have no conflicts of interest. Funding

This research received no external funding.

\section{REFERENCES}

1. Kujumgiev A, Tsvetkova I, Serkedjieva Y, Bankova V, Christov R, Popov S. Antibacterial, antifungal and antiviral activity of propolis of different geographic origin. J Ethnopharmacol. 1999;64:235-40.

2. Prabhakar AR, Balehosur DV, Basappa N. Comparative evaluation of shear bond strength and fluoride release of conventional glass ionomer with $1 \%$ ethanolic extract of propolis incorporated glass ionomer cement -invitro study. J Clin Diagn Res. 2016;10:88-91.

3. Subramaniam P, Girish Babu KL, Neeraja G, Pillai S. Does addition of propolis to glass ionomer cement alter its physicomechanical properties? An In Vitro Study. J Clin Pediatr Dent. 2017;41:62-5.

4. Kumazawa S, Hamasaka T, Nakayama T. Antioxidant activity of propolis of various geographic origins. Food Chem. 2004;84:329-39.

5. Hayacibara MF, Koo H, Rosalen PL, Duarte S, Franco EM, Bowen WH, et al. In vitro and in vivo effects of isolated fractions of Brazilian propolis on caries development. J Ethnopharmacol. 2005;101:110-5.

6. Russo A, Cardile V, Sanchez F, Troncoso N, Vanella A, Garbarino JA. Chilean propolis: antioxidant activity and antiproliferative action in human tumor cell lines. Life Sci. 2005;76:545-58.

7. Bhuvnesh A, Sarkar PA, Tomar U, Bishen KA. Antibacterial effect of propolis derived from tribal region on Streptococcus mutans and Lactobacillus acidophilus: An in vitro study. J Indian Soc Pedod Prev Dent. 2018;36:48-52.

8. Vermeersch G, Leloup G, Delmee M, Vreven J. Antibacterial activity of glass-ionomer cements, compomers and resin composites: relationship between acidity and material setting phase. J Oral Rehabil. 2005;32:368-74.

9. Davidovich E, Weiss E, Fuks AB, Beyth N. Surface antibacterial properties of glass ionomer cements used in atraumatic restorative treatment. J Am Dent Assoc. 2007;138:1347-52.

10. De Carvalho Machado J, Duque C, Pitzer de Oliveira J, Scarparo A. Effect of storage time and chlorhexidine addition on the mechanical properties of glass ionomer cements. Braz J Oral Sci. 2017;16:1-9. 
El Ghazouly et al.

11. Altunsoy M, Tanriver M, Turkan U, Uslu ME, Silici S. In vitro evaluation of microleakage and microhardness of ethanolic extracts of propolis in different proportions added to glass ionomer cement. J Clin Pediatr Dent. 2016;40:136-40.

12. Nursen T, Ozan F, Ozyurt M, Kulekci G. In vitro antibacterial effects of glass-ionomer cement containing ethanolic extract of propolis on Streptococcus mutans. Eur J Dent. 2012;6:428-33.

13. Erdem H, Ozturk F, Bilenler T, Aksakalli S, Simsek N. Antibacterial and mechanical properties of propolis added to glass ionomer cement. Angle Orthod. 2014;84:368-73.

14. Koo H, Pearson SK, Scott-Anne K, Abranches J, Cury JA, Rosalen PL, et al. Effects of apigenin and tt-farnesol on glucosyltransferase activity, biofilm viability and caries development in rats. Oral Microbiol Immunol. 2002;17:337-43.

15. Charan J, Biswas T. How to calculate sample size for different study designs in medical research? Indian J Psychol Med. 2013;35:121-6.

16. Nandana KL, Sankar AJS, Kumar MGM, Naveen K, Pranitha K, Manjula BS. Comparative evaluation of microleakag using three variables of glass-ionomer cement in primary and permanent teeth: An in vitro study. J Interdiscip Dent. 2016;6:110-5.

17. Pannucci CJ, Wilkins EG. Identifying and avoiding bias in research. Plast Reconstr Surg. 2010;126:619-25.
The effect of propolis on Microleakage and Microhardness of GIC

18. Ahluwalia P, Chopra S, Thomas AM. Strength characteristics and marginal sealing ability of chlorhexidine-modified glass ionomer cement: an in vitro study. J Indian Soc Pedod Prev Dent. 2012;30:41-6.

19. Frencken JE, Holmgren CJ. Atraumatic restorative treatment (ART) for dental caries. Nijmegen: STI Book; 1999.

20. Muangmingsuk A, Senawongse P, Yudhasaraprasithi S. Influence of different softstart polymerization techniques on marginal adaptation of Class $\mathrm{V}$ restorations. Am J Dent. 2003;16:117-9.

21. Nagaa AA,Yousef M, Ramadan R, Fayez Bahgat S, Alshawwa L. Does the use of a novel self -adhesive flowable composite reduce nanoleakage? Clin Cosmet Investig Dent. 2015;7:55-64.

22. Hossain M, Yamada Y, Nakamura Y, Murakami Y, Tamaki Y, Matsumoto K. A study on surface roughness and microleakage test in cavities prepared by Er:YAG laser irradiation and etched bur cavities. Lasers Med Sci. 2003;18:25-31.

23. Troca VB, Fernandes KB, Terrile AE, Marcucci MC, Andrade FB, Wang L. Effect of green propolis addition to physical mechanical properties of glass ionomer cements. J Appl Oral Sci. 2011;19:100-5.

24. Muangmingsuk A, Senawongse P, Yudhasaraprasithi S. Influence of different softstart polymerization techniques on marginal adaptation of Class $\mathrm{V}$ restorations. Am J Dent. 2003;16:117-9. 